\title{
Guest Atom Displacements in Silicon Cluster $\mathrm{Na}_{2} @ \mathrm{Si}_{50} \mathrm{H}_{44}$
}

\author{
Hiroyuki Takenaka* and Kazuo Tsumuraya \\ Department of Mechanical Science and System Engineering, School of Science and Engineering, Meiji University, \\ Kawasaki 214-8571, Japan
}

We present the displacements of the guest sodium atoms in double caged $\mathrm{Na}_{2} @ \mathrm{Si}_{50} \mathrm{H}_{44}$ cluster. The cluster consists of a piece of clathrate II consisting of two adjacent $\mathrm{Si}_{28}$ cages filled with guest sodium atoms, being hydrogenated in order to terminate the dangling bonds. Although in a single $\mathrm{Si}_{28} \mathrm{H}_{28}$ cage the endohedral sodium atom locates at the center, the sodium atoms in each of the double cage cluster displace about $0.06 \mathrm{~nm}$ away from each center of the cages to form a dimer between the endohedral sodium atoms. The displacements are attributed to the formation of covalent bond between the endohedral sodium atoms and the ionic bonding between the sodium atoms and the cage silicon atoms.

(Received October 17, 2005; Accepted November 10, 2005; Published January 15, 2006)

Keywords: silicon clathrate, silicon cluster, endohedral atom, density functional calculation, guest atom displacement, covalent bond, ionic bond

\section{Introduction}

Group 14 clathrates have been synthesized when the cages contain specific endohedral atoms of which elctronegative differences are large from host atoms. The endohedral atoms are alkali metallic, alkaline earth metallic, Te or I atoms. ${ }^{1-4)}$ The clathrates are expanded phases of diamond type silicon crystal. So the band gaps are greater than that of the latter. When the clathrates contain alkali metallic or alkaline earth metallic atoms, they become metallic because of the increased valence electron density released from the endohedral atoms. Then the endohedral atoms give rise to rattling motions in the encapsulated cages. So the clathrates have low thermal conductivity. The clathrates may be used for thermoelectronic devices when they give large thermo-electric power and high electric conductivity.

Several types of structures of the silicon clathrates have been found. They consist of mainly $\mathrm{Si}_{20}, \mathrm{Si}_{24}, \mathrm{Si}_{28}$, and distorted $\mathrm{Si}_{20}$ cages. The clathrate I consitsts of $\mathrm{Si}_{20}$ cages with dodecahedra (denoted $5^{12} ; 12$ pentagons) and $\mathrm{Si}_{24}$ cages with tetrakaidecahedra $\left(5^{12} 6^{2} ; 12\right.$ pentagons and two hexagons), the II consists of the $\mathrm{Si}_{20}$ cages and $\mathrm{Si}_{28}$ cages with hexakaidecahedra $\left(5^{12} 6^{4}\right)$ and the III consists of the $\mathrm{Si}_{20}$ cages and distorted $\mathrm{Si}_{20}$ dodecahedra. Among these, the clathrate II is composed of the largest $\mathrm{Si}_{28}$ cages and the smallest $\mathrm{Si}_{20}$ cages. So, the clathrate II may have the lowest thermal conductivity caused by the rattling motion in the $\mathrm{Si}_{28}$ cages. The symmetry is $F d \overline{3} m$ space group with origin at center $\overline{3} \mathrm{~m}$. The clathrate contains $34 \mathrm{Si}$ atoms per rhombohedral unit cell consisting of two $\mathrm{Si}_{28}$ cages and four $\mathrm{Si}_{20}$ cages. The structure is composed of $136 \mathrm{Si}$ atoms in simple cubic lattice containing eight $\mathrm{Si}_{28}$ cages and $16 \mathrm{Si}_{20}$ cages. The $\mathrm{Si}_{28}$ cages are connected tetrahedrally just like carbon atoms in the diamond crystal and the adjacent $\mathrm{Si}_{28}$ cages joint each other at the common hexagonal rings. The $\mathrm{Si}_{20}$ cages fill the remained room connecting each other. Figure 1 shows this situation.

Cros et al. ${ }^{5)}$ have reported that the lattice constants of the $\mathrm{Na}_{x} @ \mathrm{Si}_{136}$ clathrate II are constant up to around $x=10$ and

*Graduate Student, Meiji University (a)

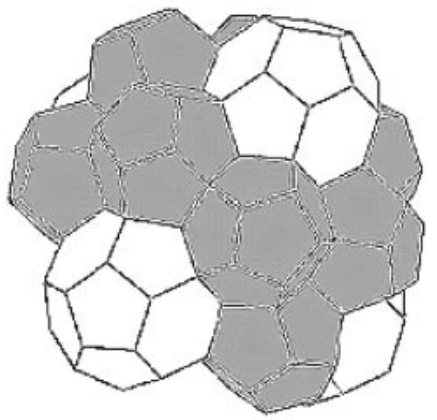

(b)

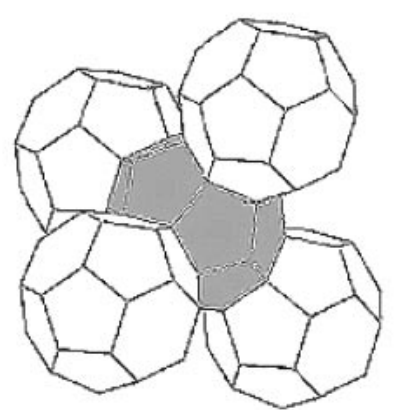

Fig. 1 (a) The coordination of connected $\mathrm{Si}_{20}$ cages around $\mathrm{Si}_{28}$ cages in clathrate II. The $\mathrm{Si}_{28}$ cages are white. Removing the shaded $\mathrm{Si}_{20}$ cages gives the tetrahedrally connected $\mathrm{Si}_{28}$ cages shown in (b). The central shaded $\mathrm{Si}_{28}$ cage is connected on the four hexagonal rings forming giant tetrahedral bonds.

increase with increasing the sodium content $x$. This means that the sodium atoms are encapsulated in the larger $\mathrm{Si}_{28}$ cages preferentially. The increase of the lattice constants is due to the encapsulation of the endohedral sodium atoms into the smaller $\mathrm{Si}_{20}$ cages. This indicates that the $\mathrm{Si}_{28}$ cage has sufficient room to accommodate the endohedral sodium atom. Demkov et $a l^{6}{ }^{6}$ have found from their density functional calculations on clathrate $\mathrm{Na}_{4} @ \mathrm{Si}_{136}$ that the guest sodium atoms move away from the center of the $\mathrm{Si}_{28}$ cages by $0.017 \mathrm{~nm}$ and the site symmetry was reduced from $T_{d}$ to $C_{3 v}$. They discussed the displacements were due to a Jahn-Teller distortion. Brunet et al. ${ }^{7)}$ have measured the displacements using extended X-ray absorption fine structure (EXAFS). They observed the displacements by $0.09 \pm 0.02 \mathrm{~nm}$ for the 
full occupation of the endohedral sodium atoms. They discussed the displacements to be attributed to Peierls or Jahn-Teller effect. Libotte et al. ${ }^{8)}$ have calculated the displacements of the endohedral sodium atoms. They found the displacements to be $0.0456 \mathrm{~nm}$ from the density functional calculation and $0.091 \mathrm{~nm}$ from a tight-binding calculation. Tournus et al. ${ }^{9)}$ have reported the guest displacements of the endohedral sodium atoms from the EXAFS measurements. The displacements were $0.1 \mathrm{~nm}$ in the $\mathrm{Si}_{28}$ cages of the clathrate $\mathrm{Na}_{2} @ \mathrm{Si}_{34}$ and $0.2 \mathrm{~nm}$ in the clathrate $\mathrm{Na}_{6} @ \mathrm{Si}_{34}$. They also calculated the displacements as $0.065 \mathrm{~nm}$ from supercell calculation of the two adjacent $\mathrm{Si}_{28}$ cages with $\mathrm{Na}_{2} @ \mathrm{Si}_{50} \mathrm{H}_{44}$ cluster using the density functional calculation.

No origin of the guest atom displacements however has been presented in the earlier papers. The present paper evaluates the guest atom displacements in the finite double caged $\mathrm{Si}_{28}$ cluster which is extracted from the clathrate II using the density functional calculation. We present the origin of the guest displacements in the cluster.

\section{Computational details}

We calculate the electronic states of the hydrogenated clusters using molecular orbitals based on a linear combination of atomic orbitals. We use a frozen core approximation for constructions of the molecular orbitals: the atomic orbitals are assigned $3 \mathrm{~s}^{1}$ for $\mathrm{Na}$ atoms, $3 \mathrm{~s}^{2} 3 \mathrm{p}^{2}$ for $\mathrm{Si}$ atoms, and $1 \mathrm{~s}^{1}$ for $\mathrm{H}$ atoms. We adopt two Slater-type functions for each orbital and the generalized gradient functional of Perdew, Burke, and Ernzerhof ${ }^{10)}$ for the exchange correlation functionals. All atoms are relaxed for the maximum magnitude of the forces to decrease less than $4.3598 \times$ $10^{-20} \mathrm{~J} / \mathrm{nm}\left(10^{-3} \mathrm{H} / \AA\right)$.

The initial coordinates of the $\mathrm{Na}_{2} @ \mathrm{Si}_{50}$ cluster are obtained from the coordinates of the clathrate II in simple cubic lattice, in which there are two adjacent $\mathrm{Si}_{28}$ cages containing two endohedral $\mathrm{Na}$ atoms. We terminate the dangling bonds of the three coordinated $\mathrm{Si}$ atoms on the cages with hydrogen atoms, which results in similar bonding states as in the crystalline state with the cluster. This termination allows the size of the cluster to shrink and allows the interatomic $\mathrm{Si}-\mathrm{Si}$ distances to decrease to those in the clathrate. In this work, we use the ADF package ${ }^{11)}$ to calculate the electronic states.

\section{Results}

First we calculate a stable position of the sodium atom in the single caged $\mathrm{Si}_{28}$ cluster hydrogen terminated, i.e. $\mathrm{Na} @ \mathrm{Si}_{28} \mathrm{H}_{28}$. When we have relaxed all the atom positions of the cluster, the endohedral sodium atom has remained at the center of the cage. Next, we calculate the cohesive energies of the cluster by displacing the sodium atom from the center of the cage. The directions are to the center of a hexagonal ring, to the center of the pentagonal ring, and to a silicon atom between them by fixing all the atom positions. The energies have been minimum at the center of the $\mathrm{Si}_{28}$ cage for the three cases.

Next, we calculate the stable positions of the sodium atoms

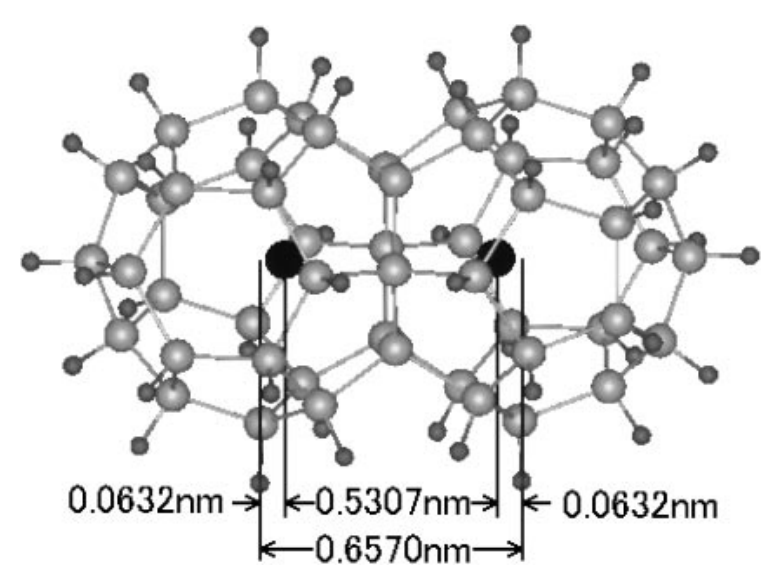

Fig. 2 The displacements of endohedral $\mathrm{Na}$ atoms in the $\mathrm{Na}_{2} @ \mathrm{Si}_{50} \mathrm{H}_{44}$ cluster. Each endohedral $\mathrm{Na}$ atom in the $\mathrm{Si}_{28}$ cages displaces by $0.0632 \mathrm{~nm}$ form the center of the cages to other side of $\mathrm{Na}$ atom, i.e. toward the center of the joint hexagonal ring.

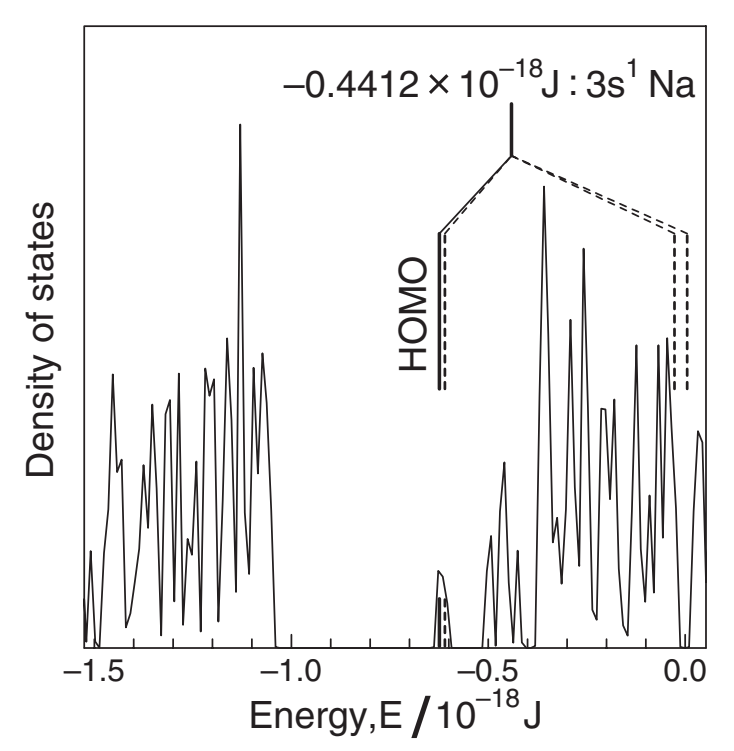

Fig. 3 The calculated total density of states of $\mathrm{Na}_{2} @ \mathrm{Si}_{50} \mathrm{H}_{44}$ cluster. We show convolution of energy levels broadened using gaussian functions with $\sigma=0.1090 \times 10^{-19} \mathrm{~J}(0.0025 \mathrm{H})$. The atomic orbital $3 \mathrm{~s}^{1}$ of an isolated sodium atom splits into the molecular orbitals with the bonding HOMO state, the antibonding unoccupied LUMO state just above the HOMO, and the unoccupied antibonding states far above the LUMO state.

in the relaxed $\mathrm{Na}_{2} @ \mathrm{Si}_{50} \mathrm{H}_{44}$ cluster, which is the double caged $\mathrm{Si}_{28}$ cluster. During the relaxation, the positions of the endohedral sodium atoms have moved by $0.0632 \mathrm{~nm}$ from the relaxed central positions in the cages toward the center of the hexagonal ring as shown in Fig. 2. The guest atoms have displaced symmetrically with respect to the ring surface. The displacements are comparable with those $0.065 \mathrm{~nm}$ by Tournus et al. ${ }^{9)}$

We have plotted the density of states in Fig. 3 in which the eigenvalues of the molecular orbitals are broadened with gaussian functions. The eigenvalue $-4.4121 \times 10^{-19} \mathrm{~J}$ $(-0.1012 \mathrm{H})$ of the $3 \mathrm{~s}$ atomic orbital of the isolated $\mathrm{Na}$ atom splits into the molecular eigenvalues. The bonding HOMO state is $-6.2380 \times 10^{-19} \mathrm{~J}(-0.1431 \mathrm{H}, \mathrm{A} 1 . \mathrm{g} 16)$ and the antibonding states are $-6.1037 \times 10^{-19} \mathrm{~J}(-0.1399 \mathrm{H}$, 

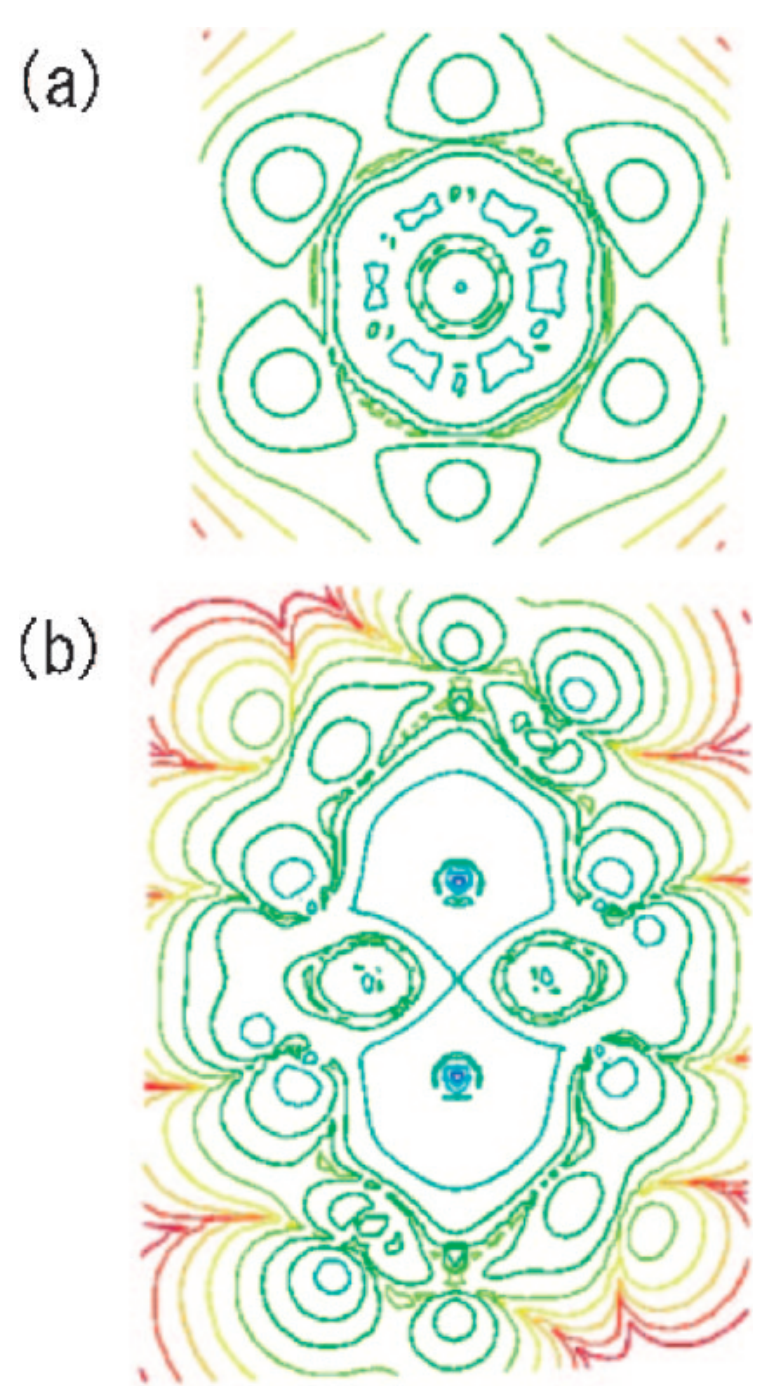

Fig. 4 The projected electron charge densities drawn with the eigenfunction of the HOMO level. (a) the density profile on the plane of joint hexagonal $\mathrm{Si}$ ring between the two $\mathrm{Si}_{28}$ cages of the cluster. (b) the density profile on the plane which intercepts both the endohedral two sodium atoms and one of silicon atom of the joint hexagonal ring. The lowest contour line corresponds to $10^{-10}$. Each contour corresponds to 12 logarithmic levels between the lowest and unity. The blue lines are higher densities than the green ones.

LUMO, A2.u15), $-0.2757 \times 10^{-19} \mathrm{~J}(-0.0063 \mathrm{H}, \mathrm{A} 1 . \mathrm{g} 23)$, $0.0467 \times 10^{-19} \mathrm{~J}(0.0011 \mathrm{H}, \mathrm{A} 2 . \mathrm{u} 24)$. There is a large forbidden gap just below the HOMO state. If the cluster contains no endohedral atom, the cluster is an insulator. The HOMO-LUMO gap is only $0.1343 \times 10^{-19} \mathrm{~J}$. Thus the cluster is easy to be removed the electrons leading to the ionized +2 state. The position of the HOMO state in the DOS is almost the same as in the clathrate II indicating that the overall electronic structure is similar to that of the clathrate II. ${ }^{12)}$

We have shown that bonding state of the sodium atoms corresponded to HOMO in Fig. 3. So, we calculate the charge density profiles using the HOMO level. The charge density on the plane of the joint hexagonal silicon ring is shown in Fig. 4(a). The each double circle on the outermost hexagon corresponds to the intersections of the $\mathrm{H}-\mathrm{H}$ bonds, where one of the hydrogen on the $\mathrm{Si}_{28}$ cage and the other is on the other

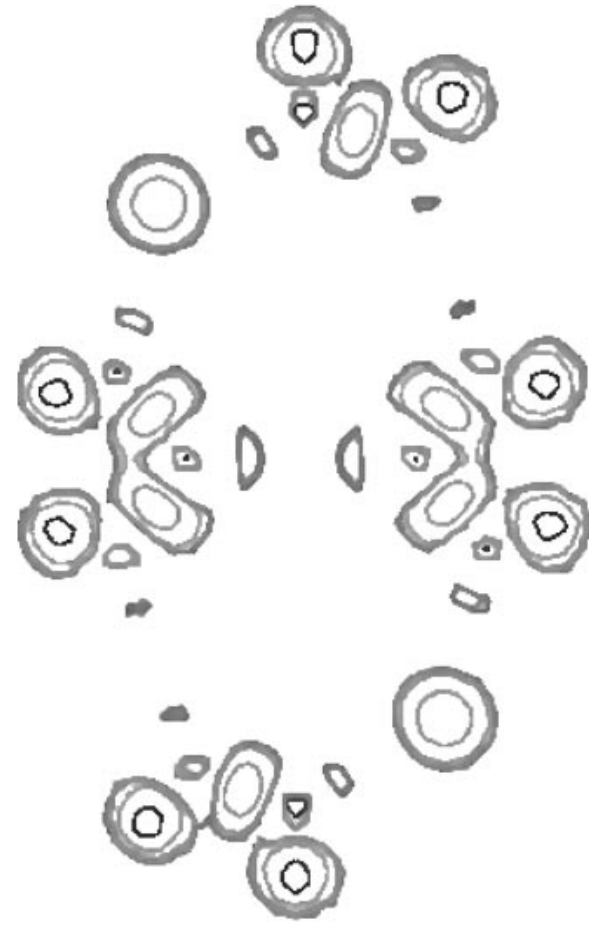

Fig. 5 The difference charge density profile between the self consistent charge density and the overlapped isolated atom densities. The intersection plane is the same as in Fig. 4(b).

$\mathrm{Si}_{28}$ cage. The inner six rectangles correspond to the covalent bonds between the hexagonal silicon ring. The silicon atoms locate between the rectangular regions. There is a finite charge density region which is circle shaped at inner of the hexagon. Figure 4(b) shows the charge density of the HOMO state which intercepts the two endohedral sodium atoms and one of the silicon atom in the hexagonal ring. There is also a finite HOMO charge density region between the two endohedral sodium atoms, indicating a covalent bonding formed between the $3 \mathrm{~s}^{1}$ electrons of each $\mathrm{Na}$ atom.

We have shown the guest atom displacements in Fig. 2. The sodium atoms in each of the double cage cluster have displaced $0.06 \mathrm{~nm}$ away from each center of the cages to form dimer between the sodium atoms. The projected charge densities of the HOMO level which corresponds to $3 \mathrm{~s}$ state of the sodium atoms showing the covalent bonding between the endohedral atoms. The displacements may be also caused by the interaction between the sodium atoms and the frame silicon atoms. To check this we calculate the difference charge density profile between the self-consistent charge density and the overlapped isolated atom densities. The density is shown in Fig. 5 with a logarithmic scale. Thus the blank region corresponds to negative charge. The regions around the endohedral sodium atoms are negative which indicates that the valence electrons have transferred from the sodium atoms to the frame silicon atoms.

To evaluate the charge transfer quantitatively, we evaluate the charge transfers of the constituent atoms. As is well known, the Mulliken's analysis depends on the basis functions used. For instance, Guerra et al., ${ }^{13)}$ ADF developers, have shown that the charge transfer of carbon atom in methane is negative for double Slater-type zeta functions 
although it is positive for the triple zeta with two sets of polarized functions. They showed that Voronoi or Hirshfeld analyses gave reasonable, comparable values with Bader charges. The charge transfers of the endohedral sodium atoms have been $0.335 \mathrm{e}$ with Voronoi analysis and $0.220 \mathrm{e}$ with Hirshfeld analysis. The received charges of the silicon atoms of the hexagonal ring have been $-0.075 \mathrm{e}$ for Voronoi analysis and $-0.033 \mathrm{e}$ for Hirshfeld analysis. The rest of the silicon $\mathrm{Si}$ atoms transfers electron as large as $0.023 \mathrm{e}$ for Voronoi analysis and $0.058 \mathrm{e}$ for Hirshfeld analysis. The electrostatic interaction exists between the sodium atoms and the frame silicon atoms, indicating the ionic bonding between them.

\section{Discussion}

The endohedral $\mathrm{Na}$ atoms in the $\mathrm{Na}_{2} @ \mathrm{Si}_{50} \mathrm{H}_{44}$ cluster have displaced from the center of the cages towards the center of the joint hexagonal ring as shown Fig. 2. We have plotted the electron charge density corresponding to the $\mathrm{Na}$ bonding state in this cluster. The bond charge density with respect to the $\mathrm{Na}-\mathrm{Na}$ atoms has appeared. Thus, this corresponds to the covalent bond between the $\mathrm{Na}$ atoms. We have presented the charge transfer using Voronoi and Hirshfeld population analyses. The charge of $0.2-0.3 \mathrm{e}$ of the valence electron of the endohedral sodium atoms has transferred to the frame silicon atoms. The rest of the electrons contribute to the covalent band between the endohedarl atoms. Although the present analysis uses the cluster model extracted from the clathrate II, both the interactions $\mathrm{Na}-\mathrm{Na}$ binding and the host-guest attraction through the charge transfer may act in the clathrate II.

\section{Conclusions}

In this paper, we have investigated the electronic states of $\mathrm{Na}_{2} @ \mathrm{Si}_{50} \mathrm{H}_{44}$ cluster to study the origin of the displacements of the endohedral atoms. Both the endohedral sodium atoms move by $0.0632 \mathrm{~nm}$ close to each other from the center of the $\mathrm{Si}_{28}$ cages. The charge density profile consists of the $3 \mathrm{~s}$ molecular orbital of sodium atom has indicated the covalent bonding charge density. Both the sodium atoms have transferred electrons by $0.2-0.3$ e to the frame silicon atoms. The displacements in this cluster are attributed to the formation of dimer between the endohedral $\mathrm{Na}$ atoms and the ionic bonds between the endohedral sodium atoms and the frame atoms.

\section{REFERENCES}

1) C. Cros: Ph. D. Thesis, No. 205, University Bordeaux (1970).

2) C. Cros, E. Reny and M. Pouchard: Mol. Cryst. Liq. Cryst. 310 (1998) 199-204.

3) S. Bobev and S. C. Sevov: J. Solid State Chem. 153 (2000) 92-105.

4) K. A. Kovnir and A. V. Shevelkov: Russian Chem. Rev. 73 (2004) 923938.

5) E. Reny, P. Gravereau, C. Cros and M. Pouchard: J. Mater. Chem. 8 (1998) 2839-2844.

6) A. A. Demkov, O. F. Sankey, K. E. Schmidt, G. B. Adama and M. O'Keeffe: Phys. Rev. B 50 (1994) 17001-17008.

7) F. Brunet, P. Melinon, A. S. Miguel, P. Keghelian, A. Perez, A. M. Flank, E. Reny, C. Cros and M. Pouchard: Phys. Rev. B 61 (2000) 16550-16560.

8) H. Libotte, J. P. Gaspard, A. Sanmiguel and P. Melinon: Europhys. Lett. 64 (2003) 757-762.

9) F. Tournus, B. Masenelli, P. Melinon, D. Connetrable, X. Blase, A. M. Flank, P. Lagarde, C. Cros and M. Pouchard: Phys. Rev. B 69 (2004) 035208-035213.

10) J. P. Perdew, K. Burke and M. Ernzerhof: Phys. Rev. Lett. 77 (1996) 3865-3868.

11) G. te Velde, F. M. Bickelhaupt, S. J. A. van Gisbergen, C. F. Guerra, E. J. Baerends, J. G. Snijders and T. Ziegler: J. Comput. Chem. 22 (2001) 931-967.

12) J. C. Conesa, C. Tablero and P. Wahnon: J. Chem. Phys. 120 (2004) 6142-6151.

13) C. F. Guerra, J. Handgraaf, E. J. Baerends and F. M. Nickelhaupt: J. Comput. Chem. 25 (2003) 189-210. 